\title{
US biologists propose launch of electronic preprint archive
}

[PARIS] An electronic challenge to traditional biomedical science journals is being planned by a group of US scientists. They propose to create an e-print archive similar to that established for physics at the Los Alamos National Laboratory in New Mexico by Paul Ginsparg in 1991.

The initiative is led by Patrick Brown, a researcher at the Howard Hughes Medical Institute at Stanford University School of Medicine, and David Lipman, director of the US National Council for Biotechnology Information (NCBI), which operates the PubMed and GenBank databases.

Under preliminary proposals, the life science e-print archives would be established at a single website. Papers could be automatically posted, archived and distributed, with authors retaining copyright over their work. Articles would either be posted without refereeing, or peer reviewed by third-party professional societies or electronic journals, and labelled as such. Signed commentaries would also be posted along with articles.

E-print archives were first established in high-energy physics as an electronic alternative to distributing preprints. But by automating the process, Ginsparg created a large free electronic journal for papers that have not been peer reviewed (see graph). This year the archive will 'publish' some 25,000 papers in astrophysics, quantum physics, condensed matter theory and computer science.

The Los Alamos archives are the primary means of first publication in many areas of physics, although many papers are later published in journals. E-print archives for cognitive science have been established by Steven Harnard at the University of Southampton, and a computer research repository for the computer sciences was recently launched by Joseph Halpern and Carl Lagoze at Cornell University, New York state.

Lipman said last week that it was too early to comment on the plans in detail. But under one proposal being discussed, the archives would benefit from direct indexing and linking via NCBI's PubMed database.

Brown says this would guarantee wide exposure, as PubMed is one of the most widely used Internet services in the biomedical sciences. It offers access to 9 million bibliographic citations from 4,000 journals in Medline, and publishes abstract information provided by publishers before it has been indexed and made available on Medline.

PubMed has hyperlinks to full text in more than 300 journals, and links molecular biology citations to protein and nucleotide sequence databases, and from one Medline

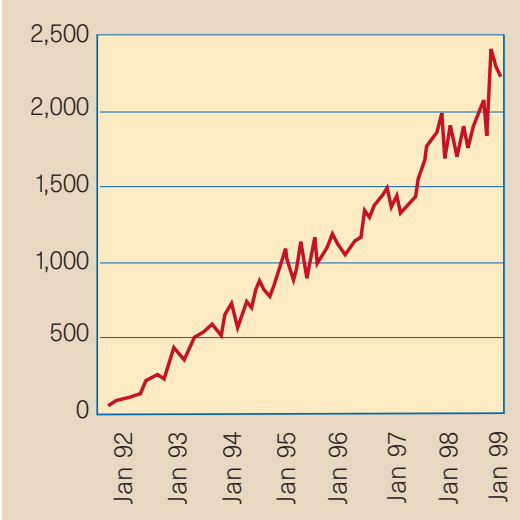

Trend setter: monthly submissions to the physics archive at Los Alamos National Laboratory have risen sharply since its launch in August 1991.

record to other related entries. The number of daily users has climbed from 35,000 to 70,000 over the past year, and PubMed now handles 1.5 million transactions daily.

Access to the proposed e-print archives would be free. The protagonists are said to be seeking funding for operating costs from the Howard Hughes Medical Institute and the US National Institutes of Health.

The initiative is likely to be welcomed in the research community. Fotis Kafatos, director of the European Molecular Biology Laboratory in Heidelberg, Germany, says: "I believe strongly that more open communication could benefit all the life sciences."

Kafatos says he would not like to see the established journals system dismantled. But he believes that an e-print archive would undermine the "scoop mentality" of some journals towards the acquisition of papers, and prompt a shift towards publishers providing greater editorial value.

The proliferation of specialized journals has compartmentalized knowledge in an artificial way, argues Kafatos. "Obviously the content of journals like Nature and Science appeals to a wide audience. But as a whole, biology is rapidly moving in the direction of dismantling barriers, and you need new ways to cut across disciplines." Electronics provides means for "more flexible organization of knowledge," he says.

Brown intends to invite leaders in the biomedical community to a workshop to discuss the proposals and encourage scientists to publish in the archive; biologists, unlike physicists, have no strong tradition of distributing e-prints.

The main obstacle facing the archive is the fear that publishing an e-print will preclude later publication in a peer-reviewed journal. Several journals (including Nature) have relatively liberal policies stating that publishing e-prints does not preclude publication, and others are expected to follow.

But some journals maintain that publishing on the Internet constitutes prior publication. Brown wrote to life-science journals this week asking them to publish "an explicit policy statement that distribution of a preprint, by means of a public electronic preprint server or Internet site, will not influence the decision of your journal to publish a paper". The letter was signed by 30 scientists including James Watson, Arthur Kornberg and Daniel Botstein.

Declan Butler

Next week: Briefing on electronic journals

\section{Indian scientist sues over spying charge}

[NEW DELHI] A rocket scientist at the Indian Space Research Organization (ISRO) in Bangalore who was falsely charged with spying five years ago has issued a $\$ 250,000$ suit against the government of Kerala state and the central government in New Delhi.

In a suit filed in a court in

Thiruvanathapuram on 2 January, Nambi Narayanan, currently director of the ISRO's advanced technology and planning department, is demanding compensation for the "mental and physical agony" he claims to have suffered at the hands of the Kerala state police and officials of the central government's intelligence bureau.

Narayanan seeks damages on ten counts, including false arrest, illegal detention for 50 days, mental and physical torture, loss of reputation, distress to his family and loss of a promotion. The court has not set a date for the hearing.

In 1994, Narayanan, then deputy director of ISRO's liquid propulsion systems centre, and three others were charged with leaking information on ISRO's cryogenic engine project to an unidentified foreign power through two women alleged to be agents of Maldives (see Nature 372, 491; 1994).

The case was dismissed as groundless by India's Supreme Court in April 1998 (see Nature 393, 6; 1998), and the two women were released in October. Narayanan says he is suing the government because the officials who "concocted the case" and tortured him had refused to admit their guilt and had not been punished.

K.S. Jayaraman 